\title{
Instabilities of Compressible Fluid Flow over a Plate
}

\author{
Sanjay Dharmavaram \\ Department of Theoretical and Applied Mechanics, Cornell University, Ithaca, NY 14853, USA \\ Anirvan DasGupta
Department of Mechanical Engineering, Indian Institute of Technology, Kharagpur, 721302, India
}

(Received 27 June 2005; revised 1 July 2006; accepted 27 April 2007)

This paper considers the linearised dynamics of flow of a compressible fluid over a plate. In particular, we study the stability of the flow, and identify the types of instability that occur. The zones of stability/instability in the Mach number-velocity ratio plane have been obtained. Two regions, namely convectively unstable and absolutely unstable, are observed to exist. Thus, the flow is always unstable. It is found that low values of Mach number and velocity ratio yield a convectively unstable flow. At high Mach number and/or velocity ratio, the system becomes absolutely unstable.

\section{INTRODUCTION}

Problems involving fluid-loaded structures are essentially of two kinds: one in which there is no mean flow of the fluid, and the other with a mean flow. These two problems are characteristically different. In the case of a structure in a static fluid, one is usually interested in modelling the fluidstructure interaction through the determination of the added mass and radiation damping parameters for the structure. There is another class of literature that deals with the response of locally excited fluid-loaded structures in the absence of a mean flow (see for example reference ${ }^{1}$ ). In such problems, the objective is to understand the spatio-temporal evolution of waves of different wave numbers when the structure is excited by a space-localised harmonic signal. On the other hand, in the case of flow of the ambient fluid past a flexible structure, there is a possibility of the structure deriving energy from the flowing fluid, and hence stability of the structure is the primary point of investigation.

Interaction of structures with flowing fluids are commonly found in engineering applications. Some examples are the wings of an aircraft in flight, a high-rise building interacting with the wind, and a high-speed rotating disc in a computer. There have been numerous studies on the stability of fluids flowing over compliant surfaces like membranes and plates. However, most of these studies have considered the fluid to be incompressible. On the other hand, the study of acoustics of vibrating structures has gained importance lately. This analysis requires the fluid to be compressible. Hence, it is of interest to study the stability of compressible fluid flow over compliant surfaces.

Fluid flow over compliant surfaces introduces considerable complications. Such problems have been studied previously, and important contributions have been made by Benja$\mathrm{min}^{2,3}$ and Landahl ${ }^{4}$. The classification of waves and their stability properties in reference ${ }^{3}$ has led to the theory of positive and negative energy waves. Cairns ${ }^{5}$ studied the effect of negative energy waves in hydrodynamic stability problems. Classically, investigations on the stability of flow over compliant surfaces study the evolution of an initial disturbance (shape and velocity) with a real wave-number on the surface.
This is essentially an initial value problem with non-local initial disturbance. However, disturbances are usually localised, and the classical stability analysis fails to capture the transient dynamics of such disturbances. For example, when a disturbance develops at a point, it may be washed downstream faster than it can spread (convective instability). On the other hand, it may spread fast enough to contaminate the whole medium (absolute instability). These concepts are discussed further in Section 3.2.

As far as locally excited fluid-loaded structures are concerned, in general it is possible that multiple solutions to a single frequency excitation exist. Such cases are handled using the concept of causality, ${ }^{6}$ which existed in the literature on plasma instability problems. ${ }^{7}$ A comprehensive summary of the methods used can be found in reference ${ }^{8}$. The instability of (incompressible) fluid flow over an elastic plate was first studied by Brazier-Smith and Scott ${ }^{9}$ largely through numerical examinations of a (quintic) dispersion relation which can be obtained analytically. They showed that at normalised flow speeds, $U$, greater than a critical value $U_{c}$, the systems become absolutely unstable. In that case, the response to any forcing diverges exponentially in time at all spatial locations of the plate. For $U<U_{c}$, the system was shown to have different characteristics for different harmonic forcing frequency ranges. In a certain range $0<\omega<\omega_{s}(U)$, the system was found to be convectively unstable, neutrally stable (with some anomalous features) for $\omega_{s}(U)<\omega<\omega_{p}(U)$, and stable and behaving essentially as in the absence of a mean flow for $\omega>\omega_{p}(U)$. An analytical solution for the above problem has been presented by Crighton and Oswell, ${ }^{10}$ where asymptotic expressions are found for $U_{c}$ and the frequencies $\omega_{s}(U)$ and $\omega_{p}(U)$. An energy equation governing the behaviour is derived. This is used to illuminate properties of the convectively unstable and neutral waves. Anomalous propagation is investigated further from the viewpoint of the theory of negative energy waves. The stability of a fluid flow over a membrane has been studied by Metcalfe. ${ }^{11}$ However, he has also considered an incompressible fluid.

Stability of elastic waves that develop in elastic structures unbounded in the streamwise direction (such as infinite plates, membranes, shells and pipes) has been considered in the 
references. ${ }^{12,13}$ In general, the interaction between the fluid and structure is non-linear, and can be handled only using numerical methods. ${ }^{14,15}$ However, the analysis is simplified considerably if one considers the linearised interaction dynamics of an inviscid fluid with a structure. With such simplifications, it is usually possible to derive the dispersion relation analytically. Instabilities have been analysed for flow over plates, ${ }^{16,17}$ membranes, shells, ${ }^{17}$ and flow through pipes. ${ }^{18}$ However, all these studies consider the fluid to be incompressible. The study of the flow of a compressible fluid over an infinite membrane can be found in the work of Dharmavaram and Dasgupta. ${ }^{20}$

The present work deals with the linear stability analysis of the flow of a compressible fluid over a plate. The dispersion relation has been obtained using the method of normal modes. The Briggs-Bers methodology has been followed to investigate the conditions of instability. A new numerical method has been proposed to take care of the complex branch cuts associated with compressible flows. Various instabilities of the flow have been identified and categorised on the Mach number-velocity ratio (non-dimensional) parameter plane. It has been observed that the compressible flow over a plate is always unstable.

\section{PROBLEM FORMULATION}

\subsection{Goveming Equations}

The situation considered in the present problem is as follows: the fluid (which is assumed to be compressible) with a mean density $\rho_{0}$, at a mean pressure $p_{0}$, is flowing past an infinite plate having linear density $\sigma_{p}$ and flexural rigidity $B$ at a constant velocity of $U$ as shown in Fig. 1. Without loss of generality, the direction of fluid flow can be assumed to be the positive $x$-axis, and the $y$-axis is perpendicular to the plate, as shown in the Fig. 1

$$
\begin{gathered}
\frac{D^{2} \tilde{p}}{D \tilde{t}^{2}}=c^{2} \nabla^{2} \tilde{p} ; \\
\frac{D \tilde{p}}{D \tilde{t}}+c^{2} \rho_{0} \nabla^{2} \tilde{\phi}=0 ; \\
\sigma_{p} \frac{\partial^{2} \tilde{\xi}}{\partial \tilde{t}^{2}}+B \frac{\partial^{4} \tilde{\xi}}{\partial \tilde{x}^{4}}=-\tilde{p}(\tilde{y}=0)+\delta(x) \tilde{F}(t) ; \\
\frac{\partial \tilde{\phi}}{\partial \tilde{y}}(\tilde{y}=0)=\left[\frac{\partial \tilde{\xi}}{\partial \tilde{t}}+U \frac{\partial \tilde{\xi}}{\partial \tilde{x}}\right],
\end{gathered}
$$

where $\tilde{p}, \tilde{\rho}$, and $\tilde{\phi}$ are the perturbed pressure, density, and velocity potential of the fluid, $\tilde{\xi}$ is the response of the plate, $\rho_{0}$, the mean density of the fluid, $\sigma_{p}$, the areal density of the plate, $c$ is the speed of sound in the fluid, and $\tilde{F}(t)$ is a time dependent line forcing. We assume that $F(t)=0$ for $t<0$ to properly enforce causality. ${ }^{9}$ We further assume that $\nabla \tilde{\phi} \rightarrow 0$ as $y \rightarrow \infty$.

Non-dimesionalising the above equations with the following substitutions,

$$
p=\frac{\tilde{p}}{p_{0}} ; \rho=\frac{\tilde{\rho}}{\rho_{0}} ; \xi=\frac{\tilde{\xi}}{L} ; x=\frac{\tilde{x}}{L} ; y=\frac{\tilde{y}}{L} ; t=\frac{\tilde{t}}{L / U} ; \phi=\frac{\tilde{\phi}}{U L},
$$

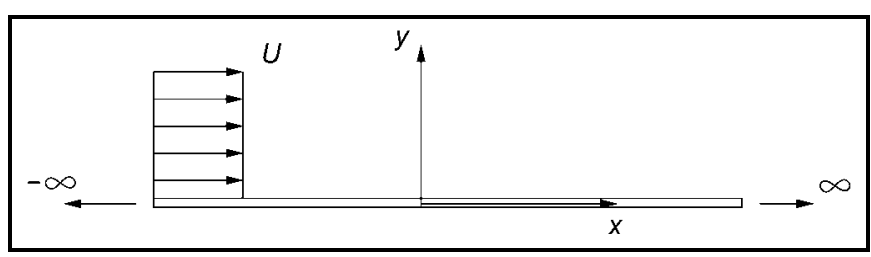

Figure 1. An infinite plate in uniform flow.

(where $L$ is a characteristic length) we have,

$$
\begin{gathered}
\frac{D^{2} p}{D t^{2}}=\frac{1}{M^{2}} \nabla^{2} p ; \\
\frac{D p}{D t}+\frac{c^{2} \rho_{0}}{p_{0}} \nabla^{2} \phi=0 ; \\
\frac{\partial^{2} \xi}{\partial t^{2}}+\left(\frac{B}{U^{2} L^{2} \sigma_{p}}\right) \frac{\partial^{4} \xi}{\partial x^{4}}=-\left(\frac{p_{0} L}{U^{2} \sigma_{p}}\right) p+\delta(x) F(t) ; \\
\frac{\partial \phi}{\partial y}(y=0)=\left(\frac{\partial \xi}{\partial t}+\frac{\partial \xi}{\partial x}\right),
\end{gathered}
$$

where $M=U / C$ is the Mach number. Next, we analyse the coupled fluid-structure system using the method of normal modes.

\subsection{The Dispersion Relation}

Let us consider the normal modes (or the Fourier modes)

where

$$
\begin{aligned}
& p=\widehat{P} e^{i\left[\left(k_{f} \cos \psi\right) x+\left(k_{f} \sin \psi\right) y-\omega t\right]} ; F=\widehat{F} e^{i(k x-\omega t)} ; \\
& \phi=\widehat{\Phi} e^{i\left[\left(k_{f} \cos \psi\right) x+\left(k_{f} \sin \psi\right) y-\omega t\right]} ; \xi=\widehat{\xi} e^{i(k x-\omega t)},
\end{aligned}
$$

$$
k=k_{f} \cos \psi,
$$

and $k$ and $k_{f}$ are the wave numbers in the plate and fluid, respectively. Then, from Eqs. (5)-(8), one obtains

$$
\begin{gathered}
\left(\omega-k_{f} \cos \psi\right)^{2}=\frac{k_{f}^{2}}{M^{2}} \\
\left(-i \omega+i k_{f} \cos \psi\right) \hat{P}-\frac{c^{2} \rho_{0}}{p_{0}} k_{f}^{2} \widehat{\Phi}=0 ; \\
\left(k^{4} a^{2}-\omega^{2}\right) \hat{\xi}=-\frac{p_{0} L}{U^{2} \sigma_{p}} \widehat{P}+\widehat{F} \\
\left(k_{f} \sin \psi\right) \widehat{\Phi}=-(\omega-k) \widehat{\xi},
\end{gathered}
$$

where $a^{2}=B / U^{2} L^{2} \sigma_{p}$ and $1 / \alpha$ will be referred to as the velocity ratio (since it is proportional to the ratio of the velocity of the fluid, to the velocity of flexural waves in the plate). Eliminating $\widehat{\Phi}$ between Eqs. (11) and (13), and using Eq. (9) yields

$$
\widehat{P}=\frac{c^{2} \rho_{0}}{i p_{0}} \frac{k_{f}}{\sin \psi} \widehat{\xi}
$$

Putting Eq. (14) back into Eq. (12) we obtain

$$
\left[\left(a^{2} k^{4}-\omega^{2}\right)^{2}+\left(\frac{c^{2} \rho_{0} L}{i U^{2} \sigma_{m}}\right) \frac{k_{f}}{\sin \psi}\right] \widehat{\xi}=\widehat{F} \Rightarrow D(k, \omega) \hat{\xi}=\widehat{F},
$$


where

$$
D(k, \omega)=\left(a^{2} k^{4}-\omega^{2}\right)+\left(\frac{c^{2} \rho_{0} L}{i U^{2} \sigma_{p}}\right) \frac{k_{f}}{\sin \psi} .
$$

Using Eqs. (9) and (10) in Eq. (16), we have, on simplification, the dispersion relation,

$$
D(k, \omega)=\left(a^{2} k^{4}-\omega^{2}\right)-\frac{\Lambda(\omega-k)^{2}}{\gamma},
$$

where $\Lambda=\rho_{0} L / \sigma_{p}$ and $\gamma^{2}=k^{2}-M^{2}(\omega-k)^{2}$. The condition that $\nabla \phi \rightarrow 0$ as $y \rightarrow \infty$ implies that $\operatorname{Re}(\gamma)>0$.

From the Fourier integral we can write, using Eq. (15), the response of the plate as

$$
\xi(x, t)=\frac{1}{4 \pi^{2}} \int_{\Gamma_{k}} \int_{\Gamma_{\omega}} \frac{\widehat{F}}{D(k, \omega)} e^{i(k x-\omega t)} d \omega d k .
$$

It is clear that the zeros of the denominator decide the nature of the solution. Therefore, the dispersion relation, $D(k, \omega)=0$, plays a central role in determining the solution characteristics, and hence, the stability of the system. The two contours of integration $\Gamma_{\omega}$ and $\Gamma_{k}$ are chosen to satisfy the causality requirement, and are discussed further in the next section.

\section{METHODOLOGY}

In this section, we briefly discuss the Briggs-Bers method and the type of instabilities. More detailed discussions can be found in the work of Brazier-Smith and Scott. ${ }^{9}$

\subsection{Briggs-Bers Method}

If $F(t)=H(t) e^{i \omega_{0} t}$, where $H(t)$ is the Heaviside function, then $\widehat{F}=i\left(\omega-\omega_{0}\right)^{-1}$ and we have from Eq. (18)

$$
\xi(x, t)=\frac{i}{4 \pi^{2}} \int_{\Gamma_{k}} \int_{\Gamma_{\omega}} \frac{e^{i(k x-\omega t)}}{D(k, \omega)\left(\omega-\omega_{0}\right)} d \omega d k .
$$

In order to satisfy the causality requirements such that $\xi=0$ for $t<0, \Gamma_{\omega}$ must pass above all the zeros of the dispersion relation for $k \in \Gamma_{k}$. Suppose that we can deform $\Gamma_{\omega}$ onto the real axis in the $\omega$ plane. We can close the $\Gamma_{\omega}$ contour in the lower half-plane, and at large time any poles in the lower half of the $\omega$ plane will give decaying waves, so we can asymptotically evaluate Eq. (19) as

$$
\xi(x, t) \sim \frac{e^{i\left(\omega_{0} t\right)}}{2 \pi} \int_{\Gamma_{k}} \frac{e^{i k x}}{D\left(k, \omega_{0}\right)} d k .
$$

This procedure fails if a pole approaches the $\Gamma_{k}$ contour in the $k$ plane as we deform the $\Gamma_{\omega}$ contour in the $\omega$ plane. If this happens, we need to indent the $\Gamma_{k}$ contour around the pole. Provided that we can keep indenting the $\Gamma_{k}$ contour we can continue to deform the $\Gamma_{\omega}$ contour. Whether a root lies above or below the indented $\Gamma_{k}$ contour determines whether it appears downstream or upstream, respectively.

However, if we cannot keep indenting the $\Gamma_{k}$ contour, then we cannot continue to deform the $\Gamma_{\omega}$ contour and the asymptotic solution in Eq. (20) is not valid. The only situation that can stop the indentation process of the $\Gamma_{k}$ contour is when two roots, starting on different sides of the $\Gamma_{k}$ contour at a large $\operatorname{Im}(\omega)$ value, merge (i.e., there is a double root) at some value of $\operatorname{Im}(\omega)$. If this merging occurs at $(\omega, k)=\left(\omega^{*}, k^{*}\right)$, the time response will be dominated by a term of the form $t^{-1 / 2} e^{i\left(k^{*} x-\omega^{*} t\right)} .^{9}$ Hence, if $\operatorname{Im}\left(\omega^{*}\right)>0$ when the merging occurs, the response will exponentially grow in time leading to instability. This idea was originally developed by Briggs ${ }^{7}$ and has since been widely used.

\subsection{Convective and Absolute Instability}

The nature of instability may be categorised as absolute instability and convective instability. An unstable flow is said to be convectively unstable if the unstable wave packet is convected away to leave the source ultimately undisturbed, as shown in Fig. 2(a). A flow becomes absolutely unstable if the unstable wave packet surrounds the source and gradually contaminates the entire medium, as depicted by Fig. 2(b). For an unstable flow that is marginally convectively/absolutely unstable, the trailing-edge of the wave packet remains at the source location.

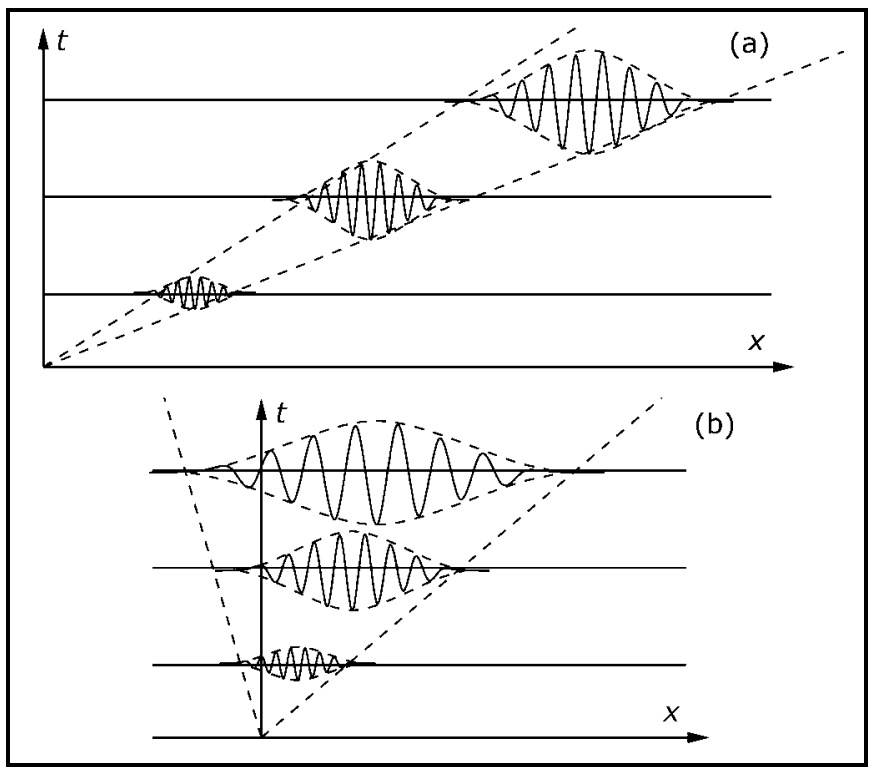

Figure 2. Convective and absolute instabilities on the $x-t$ plane.

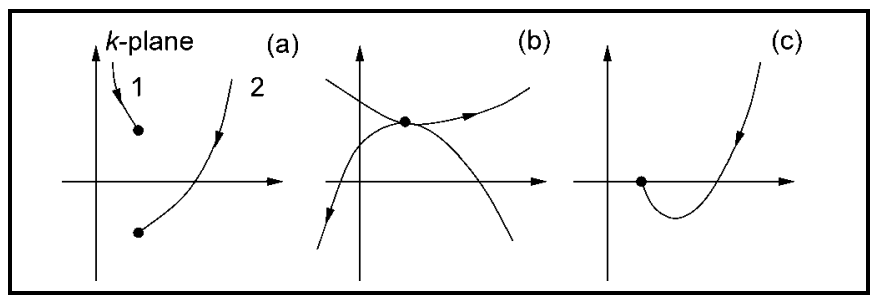

Figure 3. Schematic representation of (a) convective instability, (b) absolute instability, and (c) anomalous propagation.

Suppose that two poles come together from either side of the $k$-contour at $\omega=\omega_{p}$ corresponding to a double root of $D\left(k, \omega_{p}\right)=0$ at $k=k_{p}$. It has been proved by Briggs ${ }^{7}$ that the condition for absolute instability is $\operatorname{Im}\left(\omega_{p}\right)>0$, as shown in Fig. 3(b).

As $\operatorname{Im}(\omega)$ is reduced to zero, if there exists a pole in the $k$-plane which starts off in the upper half-plane and ends up in the lower half-plane, then the flow becomes convectively unstable, Fig. 3(a). If the pole moves from the upper halfplane to the lower half-plane and again moves back onto the real $k$-axis as $\operatorname{Im}(\omega)$ goes to zero (see Fig. 3(c)), the flow is still stable, but exhibits anomalous propagation. ${ }^{9}$ 


\section{STABIUTY ANALYSIS}

The task now is to investigate the stability of the coupled fluid-plate system by looking at the signatures of stability/instability discussed in the previous section. We will primarily rely on numerical methods for this analysis.

\subsection{Numerical Method}

It can be seen from Eq. (17) that the dispersion relation has a branch cut introduced by the equation $\gamma^{2}=k^{2}-M^{2}(\omega-k)^{2}$. For $M \neq 0$ the branch cut is complicated and the method of tracking roots on either side of the branch cut as used by Brazier-Smith and Scott fails. ${ }^{9}$ The branch cut problem may be circumvented by using the numerical scheme proposed in reference ${ }^{20}$ and presented in the following for completeness.

The obtained dispersion relation is first squared, that is,

$$
\tilde{D}(k, \omega)=\left(a^{2} k^{2}-\omega^{2}\right)^{2}-\frac{\Lambda^{2}(\omega-k)^{4}}{\left(k^{2}-M^{2}(\omega-k)^{2}\right)}=0 .
$$

However, this squaring introduces solutions not admissible by the original equation. Hence, the solutions must be screened before being accepted. The following scheme has been proposed. Each solution obtained from the above Eq. (21) is substituted in Eq. (17) and solved for $\gamma$. If $\operatorname{Re}(\gamma)>0$ then the solution is accepted, otherwise it is rejected.

To determine the transition boundary between convective and absolute instabilities (discussed in the next section) the conditions ${ }^{19}$

$$
\tilde{D}(k, \omega)=\frac{\partial \tilde{D}(k, \omega)}{\partial k}=\frac{\partial^{2} \tilde{D}(k, \omega)}{\partial^{2} k}=0
$$

are used. In this numerical computation also, the unwanted roots are rejected using the scheme mentioned above.

\subsection{Results and Disc ussions}

With the methodology elucidated in the previous section, results for the problem considered above are presented in this section.

First, we selected two suitable non-dimensional parameters namely, the Mach number $M$, and the velocity ratio $1 / a$ and with which we studied the stability properties of the system. These two non-dimensional parameters clearly reflected the fluid and the plate properties. The parameter $1 / a$ is similar to $U$ used in the work of Brazier-Smith and Scott, ${ }^{9}$ and so it is a convenient parameter for comparison. Our objective is now to determine the instability zones and types on the $M-1 / a$ parameter plane.

Determination and characterisation of the zones of stability/instability in the two-dimensional $M-1 / \alpha$-plane is an extremely difficult task. However, this task can be given a logical direction by first plotting the curves of neutral stability for different values of the non-dimensional parameters. A neutral stability curve is obtained by solving the dispersion relation Eq. (21) and using the proposed scheme (see Section 4.1) for positive real values of $k$ and $\omega$.

We first plotted the curve of neutral stability low values of $M$ and $1 / \alpha$. For example, Fig. 4 shows the neutral stability curve for $M=0.3$ and $1 / \alpha=0.05$. As indicated, there are three distinct regions in the figure, namely I, II, and III. In re- gions I and III, there is only one positive real root $k$ for a real value of $\omega$; while in region II, there are three positive real roots $k$ for a real value of $\omega$.

Next, following the Briggs-Bers methodology, we investigate the movement of roots on the $k$-plane when we introduce an imaginary part of $\omega$, which is varied keeping the real part fixed. For example, with $\operatorname{Re}(\omega)=0.03$ fixed (which corresponds to region I in Fig. 4), reducing $\operatorname{Im}(\omega)$ from a high positive value to zero, yields the root movements in $k$-plane, as shown in Fig. 5. It may be noted that there are four branches, each representing the locus of a root of $k$ of the dispersion relation as $\operatorname{Im}(\omega)$ is reduced to zero. As $\omega \rightarrow 0$, all the curves converge onto the real $k$-axis. As $\operatorname{Im}(\omega) \rightarrow 0$, branches 2 and 3 form a complex conjugate pair, and branch 4 converges onto the positive real axis, as indicated above. A magnified view of the complex conjugate pair is shown in Fig. 6. As $\operatorname{Re}(\omega)$ is increased and the above procedure repeated, the two complex conjugate roots coalese on the $\operatorname{Re}(k)$-axis, as shown in Fig. 7, there by giving two positive real roots for $k$, as $\operatorname{Im}(\omega) \rightarrow 0$. These two roots correspond to the point $A$ in Fig. 4 where there are two repeated roots of $k$ for $\operatorname{Re}(\omega)=$ 0.04515 .

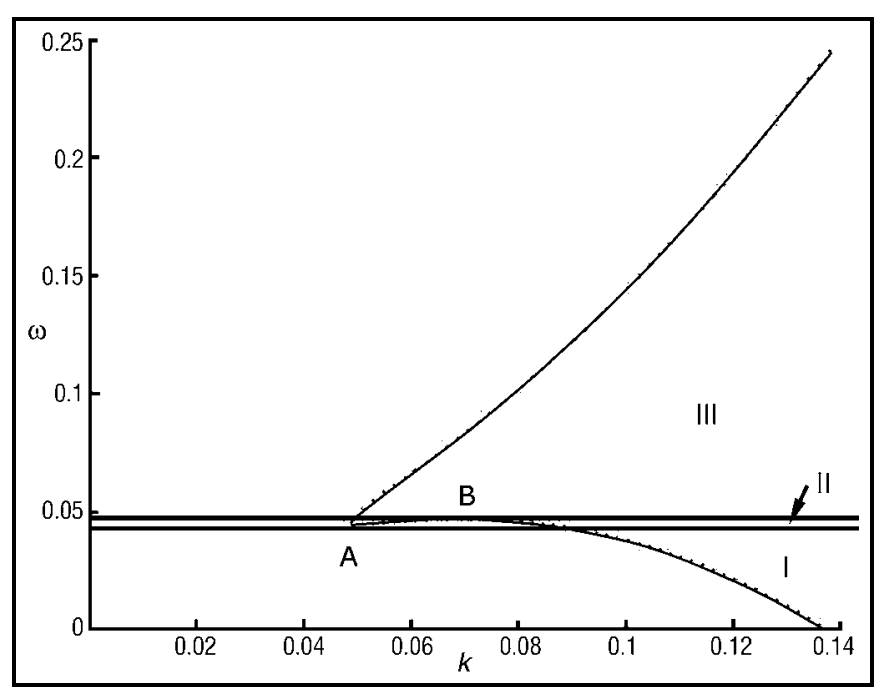

Figure 4. Neutral stability curve for $M=0.3,1 / a=0.05, \Lambda=1$.

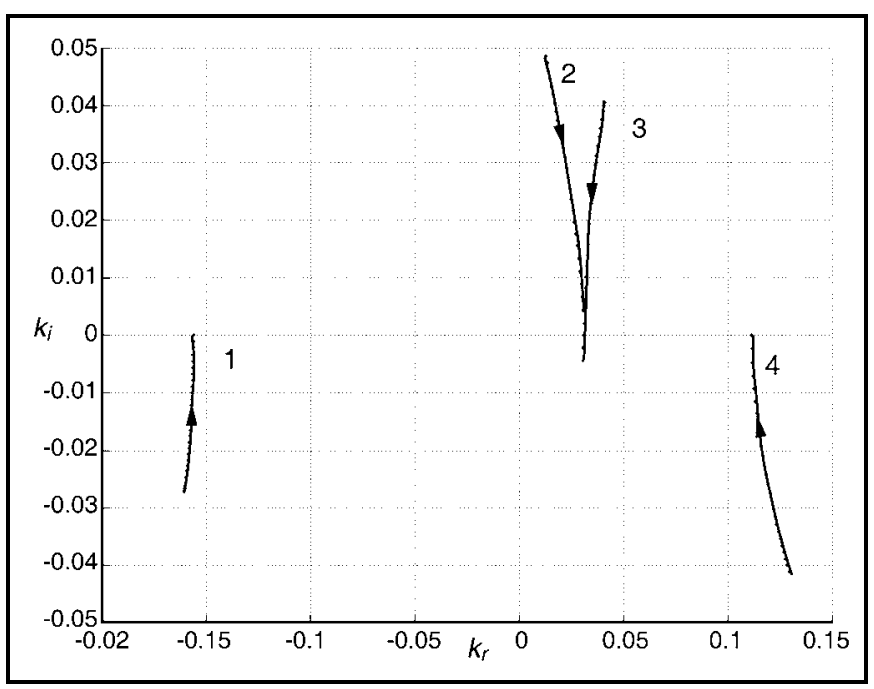

Figure 5. Locus of roots in $k$-plane as $\operatorname{Im}(\omega) \rightarrow 0, M=0.3,1 / a=0.05$, $\Lambda=1$, and $\operatorname{Re}(\omega)=0.03$. 


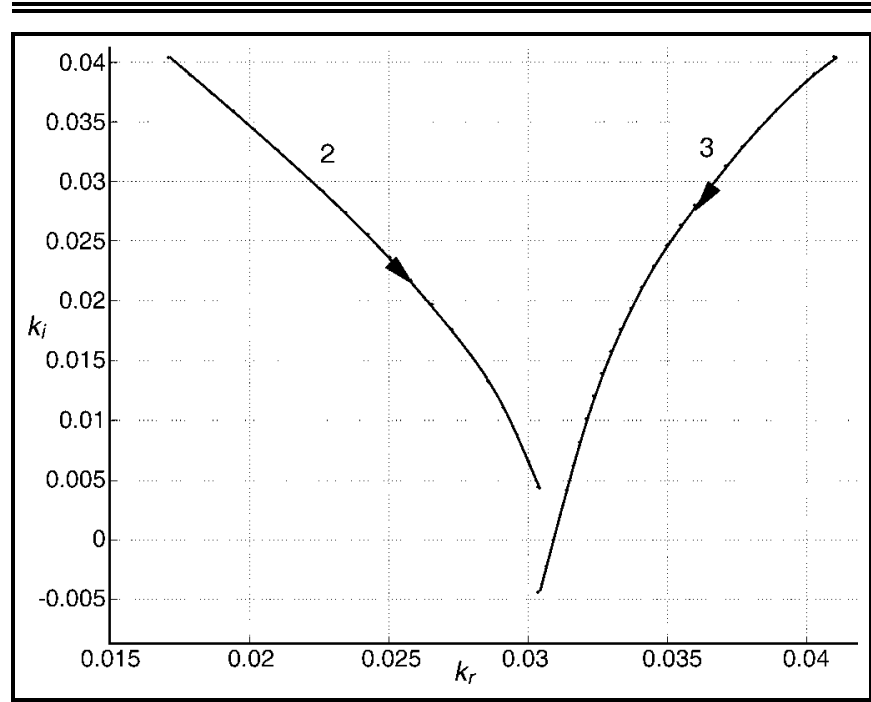

Figure 6. Magnified view of roots 2 and 3 of Fig. 5.

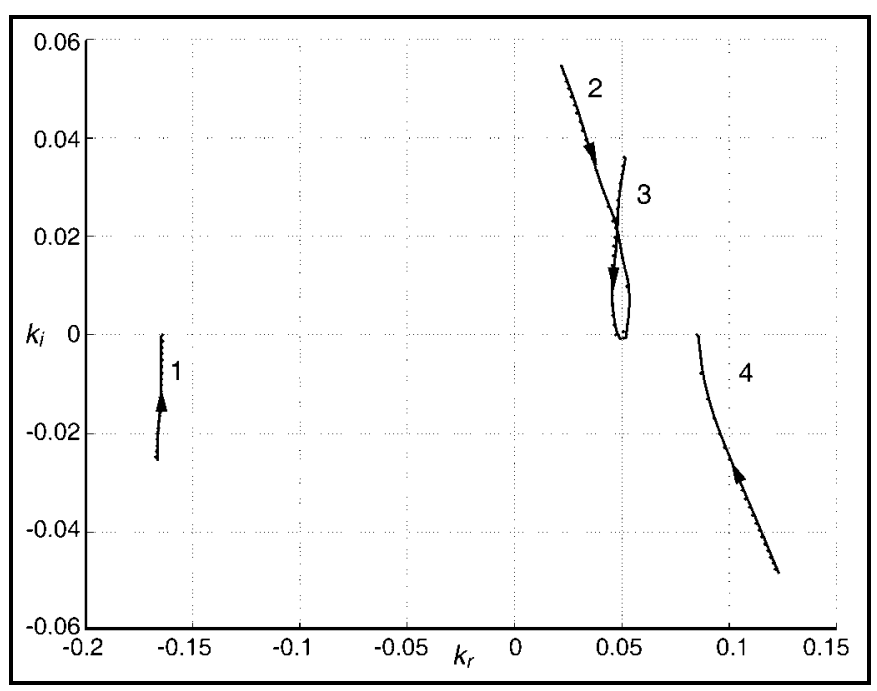

Figure 7. Locus of roots in $k$-plane as $\operatorname{Im}(\omega) \rightarrow 0, M=0.3,1 / a=0.05$, $\Lambda=1$, and $\operatorname{Re}(\omega)=0.04515$.

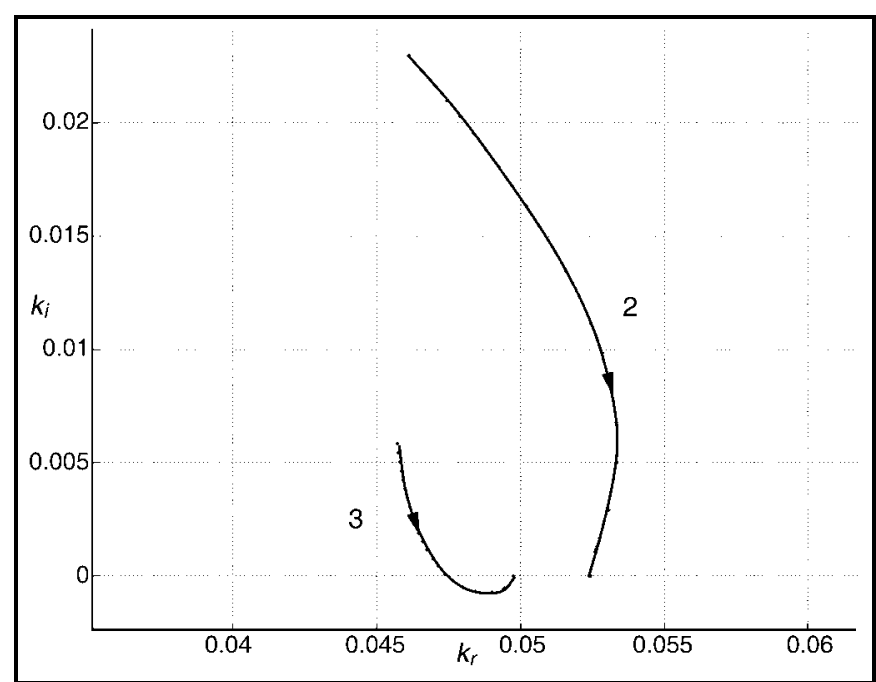

Figure 8. Magnified view of the complex conjugate root pair 2 and 3 for $\operatorname{Im}(\omega) \rightarrow 0, M=0.3,1 / \alpha=0.05, \Lambda=1$, and $\operatorname{Re}(\omega)=0.0453$.

For values of $\operatorname{Re}(\omega)$ in the region II, for example, for $\operatorname{Re}(\omega)=0.0453$, the branches 2,3 , and 4 all meet the real axis as $\operatorname{Im}(\omega) \rightarrow 0$. Thus, there exists three positive real roots of $k$ for real values of $\omega$, as already noted in Fig. 4. A magni- fied view of the branches 2 and 3 has been shown in Fig. 8. Observe that the branch 3 moves from the upper half-plane to the lower half-plane, and again moves back onto the real $k$-axis as $\operatorname{Im}(\omega)$ is reduced monotonically to zero. This is the signature of anomalous propagation. ${ }^{\mathbf{1 0}}$

As $\operatorname{Re}(\omega)$ is further increased up to the value corresponding to point B (i.e., $\operatorname{Re}(\omega)$ is 0.047353 ) in Fig. 4, the branch 2 merges with 4 as $\operatorname{Im}(\omega) \rightarrow 0$. This is shown in Fig. 9.

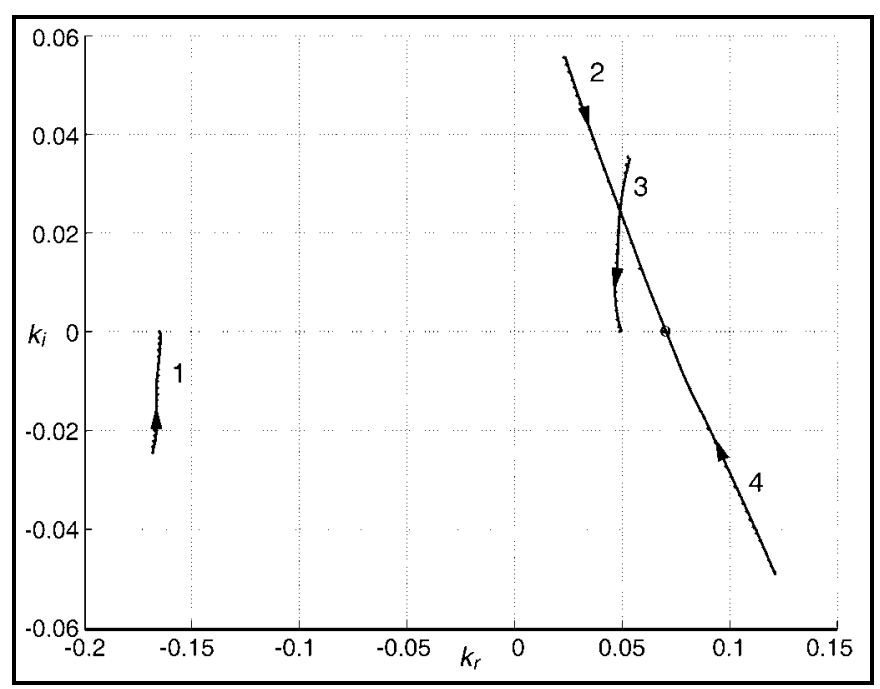

Figure 9. Locus of roots in $k$-plane as $\operatorname{Im}(\omega) \rightarrow 0, M=0.3,1 / a=0.05$, $\Lambda=1$, and $\operatorname{Re}(\omega)=0.047353$.

For values of $\operatorname{Re}(\omega)$ in region III in Fig. 4, the branches 2 and 4 form a complex conjugate pair, while 3 converges onto the real axis as $\operatorname{Im}(\omega) \rightarrow 0$, there by giving only one positive real root of $k$ for real values of $\omega$ in this region.

For large values of $M$ and/or $1 / \alpha$, analyses similar to the cases discussed above can be repeated. However, some different conclusions are obtained for large values of these parameters. Let us consider a case with $1 / \alpha=0.12$, and $M=0.4$. In Fig. 10, we have presented the root loci on the $k$-plane. When $\operatorname{Im}(\omega)$ goes to zero from a high positive value, and $\operatorname{Re}(\omega)=0.0927$ is held fixed. As $\operatorname{Im}(\omega) \rightarrow 0$ there appears only one positive real root of $k$. However, at a positive value of $\operatorname{Im}(\omega)$, there is a pinch between the two root branches 3 and 4, observed in Fig. 10. This represents absolute instability. ${ }^{9}$ This qualitative behaviour of the root loci is obtained for high values of $M$ as well.

From the above results, it is clear that for low values of the $M$ and $1 / \alpha$ we have convective instability; while for high values of any one of the parameters there is absolute instability. In order to determine the transition boundary from convective to absolute instability, we have to solve Eq. (22). The values of $M$ and $1 / \alpha$ for which the pinch occurs on the $\operatorname{Re}(k)$-axis correspond to points on the transition boundary of convective and absolute instability. ${ }^{9}$ The resulting curve plotted on the $M-1 / a$ parameter plane is shown in Fig. 11 . The curve separates the parameter plane into two zones: convectively unstable (CI) and absolutely unstable (AI), as indicated. The behaviour of the roots in the $k$-plane is qualitatively similar in each region. It may be observed from the figure that as $M \rightarrow 0$, the transition between convective and absolute instabilities occurs at $1 / \alpha=0.074$, which has also been reported by Brazier-Smith and Scott ${ }^{9}$ in their work on incompressible flow over a plate. Also, it may be noted that, unlike 
a flow on a membrane, ${ }^{20}$ no zone of stability is present. Thus, the flow of a compressible fluid over a plate is always unstable. It was observed that the length scale parameter $\Lambda$ had no effect on the zones of instability, although the loci of the roots were different.

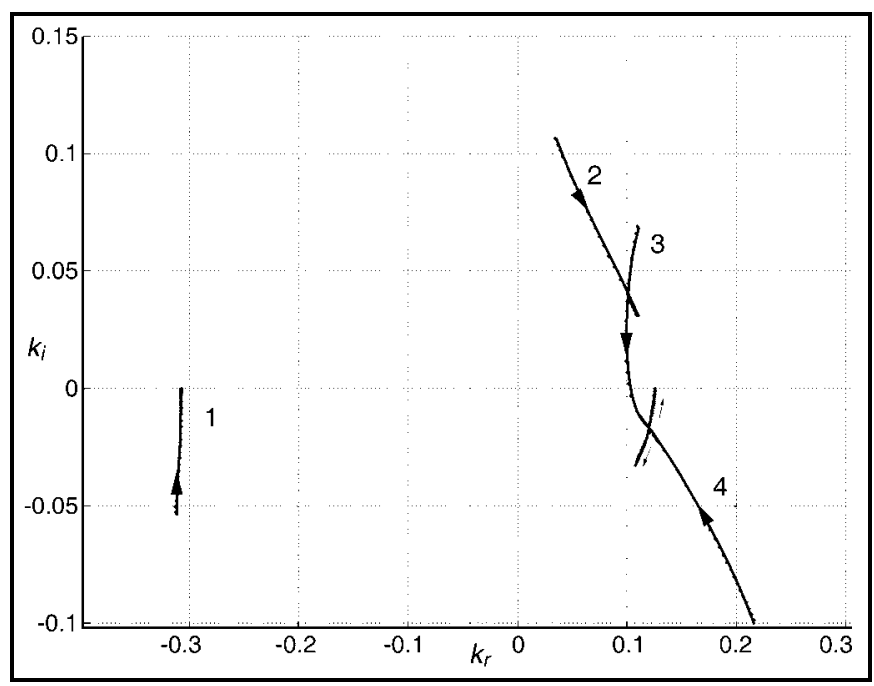

Figure 10. Locus of roots in $k$-plane as $\operatorname{Im}(\omega) \rightarrow 0, M=0.4,1 / a=0.05$, $\Lambda=1$, and $\operatorname{Re}(\omega)=0.0927$. The pinch in the complex $k$-plane occurs for $\omega^{*}=0.0927+0.0029 i, k^{*}=0.1200-0.0171 i$.

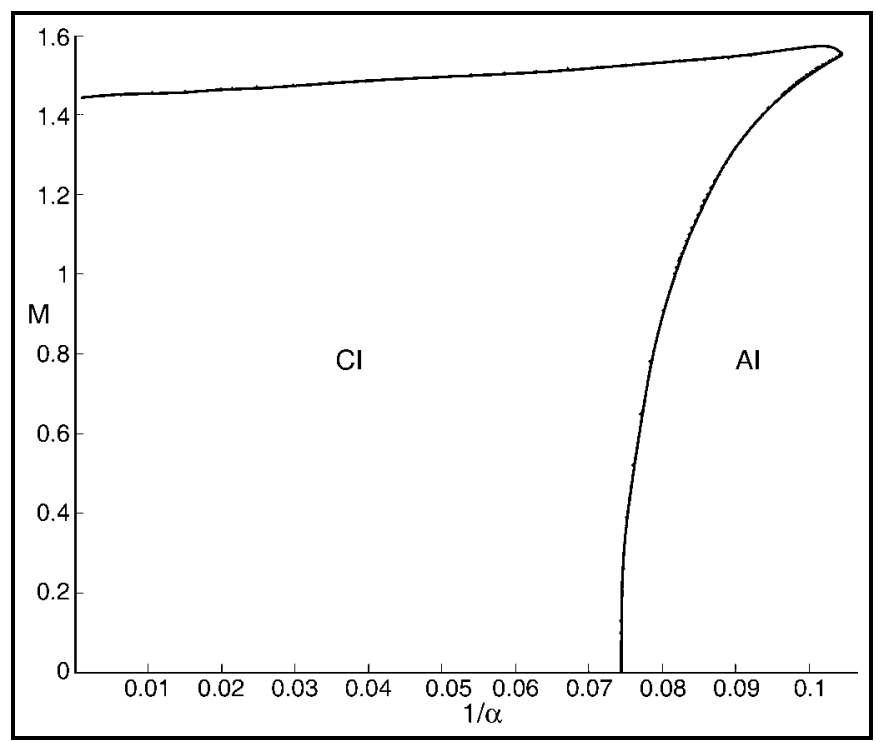

Figure 11. Curve of transition from convective instability (CI) to absolute instability (AI) (curve of marginal absolute instability), and zones showing various regions of instability.

\section{CONCLUSIONS}

The stability analysis and classification of the kinds of instabilities occurring in compressible fluid flow over a plate has been presented in this paper. It is observed that two distinct behaviours exist, convectively unstable and absolutely unstable depending on the values of the Mach number $M$ and the velocity ratio $1 / \alpha$. The system is convectively unstable for low values of $M$ and $1 / \alpha$, and absolutely unstable for high values of any one of the parameters. The plate is found to be always unstable as opposed to the case of compressible fluid flow over a membrane where a region of neutral stability was found in reference. ${ }^{20}$ The convectively unstable zone broadens as the Mach number is increased, as can be inferred from the curve of marginal absolute instability.

\section{REFERENCES}

1 Crighton, D. G. The 1988 Rayleigh medal lecture: fluid-loading - The interaction between sound an vibration, J. of Sound and Vib., 133, 1-27, (1989).

2 Benjamin, T. B. Effects of a flexible boundary on hydrodynamic stability, J. of Fluid Mech., 9, 513-532, (1960).

3 Benjamin, T. B. The threefold classification of unstable disturbances in flexible surfaces bounding inviscid flows, J. of Fluid Mech., 16, 436-450, (1963).

4 Landahl, M. T. On the stability of a laminar incompressibleboundary layer over a flexible surface, J. of Fluid Mech., 13, 609-632, (1961).

5 Cairns, R. A. The role of negative energy waves in some instabilities of parallel flows, J. of Fluid Mech., 92, 1-14, (1979).

6 Huerre, P. and Monkewitz, P. A. Absolute and convective instabilities in free shear layers, J. of Fluid Mech., 159, 151-168, (1985).

7 Briggs, R. J. Electron-stream interaction with plasmas, Monograph No. 29, MIT Press, Cambridge, Massachusetts, (1964).

8 Huerre, P. Open shear flow instabilities, Chapter 4, Perspectives in fluid dynamics, Eds. Batchelor, G. K., Moffatt, H. K., and Worster, M. G., Cambridge university Press, (2002).

9 Brazier-Smith, P. R. and Scott, J. F. Stability of fluid flow in the presence of a compliant surface, Wave Motion, 6, 547-560, (1984).

10 Crighton, D. G. and Oswell, J. E. Fluid loading with mean flow - I: Response of an elastic plate to localized excitation, Philosophical Transactions: Physical Sciences and Engineering, 1639 (335), 557-592, (1991).

11 Metcalfe, P. D. Localization and delocalization on fluid-loaded structures, PhD dissertation, University of Cambridge, (2002).

12 Walsh, C. Flutter in one-dimensional collapsible tubes, J. of Fluids and Structures, 9, 393-408, (1995).

${ }^{13}$ Huang, L. Reversal of Bernoulli effect and channel flutter, J. of Fluids and Structures, 12, 131-151, (1998).

${ }^{14}$ Lucey, A. and Carpenter, P. A numerical simulation of the interaction of a compliant wall and an inviscid flow, J. of Fluid Mech., 234, 121-146, (1992).

15 Wipler, O. and Ehrenstein, U. Numerical simulation of linear and non-linear disturbance evolution in boundary layer with compliant walls, J. of Fluids and Structures, 14, 157-182, (2000).

${ }^{16}$ Carpenter, P. W. and Garrad, A. D. The hydrodynamic stability over Kramer-type compliant surfaces. Part 2. Flow-induced surface instabilities, J. of Fluid Mech., 170, 199-232, (1986).

${ }^{17}$ Peake, N. On the behaviour of a fluid-loaded cylindrical shell with mean flow, J. of Fluid Mech., 338, 387-410, (1997).

${ }^{18}$ Langre, de E. and Ouvrard, A. E. Absolute and convective bending instabilities in fluid-conveying pipes, J. of Fluids and Structures, 13, 663-680, (1999).

19 Langre, de E. Absolutely unstable waves in inviscid hydroelastic systems, J. of Sound and Vib., 256 (2), 299-317, (2002).

${ }^{20}$ Dharmavaram, S. and Dasgupta, A. Instabilities of compressible flow over a membrane, J. of Mechanical Engineering Science, in press (2007). 